\title{
Impact of lifestyle on health-related quality of life among young university students: a cross-sectional study
}

\section{Guillermo García-Pérez-de-Sevilla', Enrique Alonso Pérez-Chao", Helios Pareja-Galeano"', Eva María Martínez-Jiménezv,} Marta de-la-Plaza-San-Frutos ${ }^{\vee}$, Beatriz Sánchez-Pinto-Pinto ${ }^{\mathrm{VI}}$, Carlos Romero-Morales ${ }^{\mathrm{VII}}$

Universidad Europea de Madrid, Spain

IPT. Physiotherapist and Professor, Department of Physiotherapy, Faculty of Sport Sciences, Universidad Europea de Madrid, Villaviciosa de Odón, Madrid, Spain.

(D) https://orcid.org/0000-0002-2689-1767

"PT. Physiotherapist and Professor, Department of Physiotherapy, Faculty of Sport Sciences, University Alfonso X, Villanueva de la Cañada, Spain.

(D) https://orcid.org/0000-0002-0228-5084

I'PT. Physiotherapist and Professor, Department of Physical Education, Sport and Human Movement, Autonomous University of Madrid, Madrid, Spain

(D) https://orcid.org/0000-0002-5780-2712

IvPT, PhD. Professor, Department of Physiotherapy, Facultad de Enfermería, Fisioterapia y Podología, Universidad Complutense de Madrid, Madrid, Spain

(D) https://orcid.org/0000-0003-0926-5200

vPT, PhD. Physiotherapist and Professor, Department of Physiotherapy, Faculty of Sport Sciences, Universidad Europea de Madrid, Villaviciosa de Odón, Madrid, Spain

(D) https://orcid.org/0000-0002-9138-9570

"MD. Physician, Physical Medicine and Rehabilitation Unit, Hospital Universitario Puerta de Hierro Majadahonda, Majadahonda, Spain.

(D) https://orcid.org/0000-0001-6444-8398

VIIPT, PhD. Physiotherapist, Department of Physiotherapy, Faculty of Sport Sciences, Universidad Europea de Madrid, Villaviciosa de Odón, Madrid, Spain.

(D) https://orcid.org/0000-0001-6598-829X

KEY WORDS (MeSH terms):

Quality of life.

Public health.

Lifestyle.

Students.

AUTHORS' KEY WORDS:

Health related quality of life.

Disease prevention.

Community health.

Physical activity.

\begin{abstract}
BACKGROUND: Lifestyle is strongly involved in the pathogenesis and progression of noncommunicable diseases, and has a great impact on quality of life. The goal of the present study was to analyze the lifestyle and body composition (BC) of young university students during the pandemic, and their relationship with health-related quality of life ( $\mathrm{HrQoL})$.

DESIGN AND SETTING: Observational cross-sectional study conducted in the Universidad Europea de Madrid, Spain.

METHODS: A total sample of 56 healthy university students was recruited. Activity, sitting time, adherence to Mediterranean diet and BC were measured.

RESULTS: Regarding BC, only $5 \%$ and $10.7 \%$ of the subjects had health risk values for waist circumference and waist-to-height ratio, respectively. The mean daily sitting-time was 8.26 hours, while $19.64 \%$ of the subjects spent $\geq 10$ hours per day sitting. $92.86 \%$ of the subjects complied with the World Health Organization 2020 physical activity recommendations. The mean PREDIMED score was 7.41, while 51.8\% of the subjects had low adherence to the Mediterranean diet. Regarding HrQoL, 22 subjects (39.2\%) and 26 subjects (46.4\%) were in the lowest quintile of physical component summary and mental component summary, respectively, according to the reference values for their age range. There was a negative correlation between physical function and sitting time $(r=-0.38)$.

CONCLUSIONS: There were high levels of sedentary behavior and low HrQoL values, with a negative moderate correlation between these variables. The findings from the present study especially highlight the importance of implementing public health programs targeting reduction of sitting time among university students.
\end{abstract}

\section{INTRODUCTION}

Lifestyle, through epigenetic mechanisms, is strongly involved in the pathogenesis and progression of noncommunicable diseases (NCDs), ${ }^{1}$ and has a great impact on quality of life. ${ }^{2}$ In this order, physical inactivity and obesity are, respectively, the two lifestyle risk factors most associated with the development of NCDs. ${ }^{3}$ Physical inactivity is the fourth largest risk factor for mortality worldwide, ${ }^{3}$ and it is associated with the comorbidities of overweight and obesity. People with these two conditions account for $27 \%$ of type 2 diabetes mellitus (T2DM) cases worldwide and $30 \%$ of ischemic heart disease cases. ${ }^{4}$ Consequently, the World Health Organization (WHO) has instituted the target of reducing the prevalence of physical inactivity by $15 \%$ worldwide by $2030 .^{5}$

Every extra hour of daily sedentary behavior has a negative impact on health. ${ }^{6}$ Compared with subjects who sit for 6 hours a day, those who sit for 8 hours have a 14\% higher cardiovascular risk and those who sit for 10 hours a day, $29 \% .^{7}$ There is strong evidence that individuals who maintain sedentary behavior over time have greater all-cause mortality, as well as several negative health-related outcomes. This is more pronounced among physically inactive people, given that physical activity attenuates mortality risk. Higher physical activity levels among highly sedentary individuals are thus required. ${ }^{8}$

The possible pathways to negative health-related outcomes facilitated by a sedentary lifestyle include promotion of increased oxidative stress. This is a strong precursor of endothelial dysfunction and gives rise to greater release of free fatty acids in the bloodstream, thus favoring 
development of cardiovascular diseases (CVDs). ${ }^{9}$ In addition, a sedentary lifestyle causes insulin resistance ${ }^{10}$ and promotes accumulation of visceral fat. ${ }^{11,12}$

Previous studies have reported that each additional hour of sedentary time is associated with greater gain in body mass index (BMI) and waist circumference (WC). ${ }^{8}$ A sedentary lifestyle can lead to development of obesity, which is the second highest risk factor for lifestyle-related premature death, after physical inactivity. ${ }^{13}$ Moreover, overweight and obesity cause insulin resistance ${ }^{14,15,16}$ and promoting deposition of ectopic lipids or visceral fat in different organs. ${ }^{17}$ This interferes with the normal function of organs, which thus increases the risk of many NCDs, especially CVD and T2DM. ${ }^{18}$ Physical activity is essential for achieving energy balance and weight control, thereby enabling maintenance of healthier body composition (BC) with an acceptable ratio of fat and muscle. ${ }^{19}$ It also protects against numerous NCDs, ${ }^{20,21}$ because it has a systemic dose-dependent anti-inflammatory effect. ${ }^{6,22,23}$

Regarding nutritional habits, adherence to the Mediterranean diet has been shown to have significant inverse associations with all-cause mortality ${ }^{24}$ and with the prevalence of CVD and T2DM, which are related to the anti-inflammatory properties of this diet. ${ }^{25,26}$ Severe acute respiratory syndrome coronavirus 2 (SARS-CoV-2) was discovered in Wuhan, Hubei province, China, in a pneumonia epidemic in January $2020 .{ }^{27}$ On January 30,2020 , it was declared by WHO to be a global health emergency ${ }^{28}$ and, since then, the virus has spread throughout the world, causing more than a million deaths.

The coronavirus disease 2019 (COVID-19) pandemic has affected and overwhelmed healthcare systems in unprecedented ways. Consequently, pending mass and effective vaccination, social distancing is the main preventive measure against SARS-CoV-2. Social distancing implies massive reorganization of society and lifestyles..$^{29}$ During the period of confinement experienced between March and May 2020 in many countries, the number of people who practiced physical activity decreased by approximately $30 \%{ }^{30}$ and the number of hours spent sitting increased by $28.6 \% .{ }^{31}$ However, adherence to the Mediterranean diet was not significantly affected. ${ }^{32}$

NCDs generally develop in the elderly population, but lifestyle behaviors become established during young adulthood, when individuals do not perceive that an unhealthy lifestyle implies a higher health risk.

There is great variability in the dietary patterns of university students, in part because there are many different questionnaires assessing the adherence to the Mediterranean diet. ${ }^{33}$ Generally, university students have a low adherence to the Mediterranean diet. ${ }^{34,35}$ Regarding physical activity levels, university students normally are highly active, but also report high amounts of sedentary behavior. ${ }^{36,37}$ Students with higher levels of physical activity usually report greater levels of health-related quality of life (HrQoL), especially regarding the mental component summary (MCS). ${ }^{38-40}$
Further research on young adulthood is needed in order to ascertain the impact of lifestyle on HrQoL in young populations.

\section{OBJECTIVE}

We aimed to analyze the lifestyle and $\mathrm{BC}$ of young university students during the pandemic, and their relationship with HrQoL. We hypothesized that physical activity levels, sedentary behavior, unhealthy $\mathrm{BC}$ and poor adherence to the Mediterranean diet would have moderate negative associations with HrQoL.

\section{METHODS}

\section{Design}

An observational cross-sectional study was developed, following the STROBE (strengthening the reporting of observational studies in epidemiology) guidelines, between October and November 2020 at Universidad Europea de Madrid, in Spain.

\section{Settings and participants}

A total sample of 56 healthy young students was recruited at Universidad Europea de Madrid: age $23.5 \pm 3.4$ years; height $175 \pm 8.1 \mathrm{~cm}$; and body mass $68.3 \pm 10.2 \mathrm{~kg}$. The participants were recruited via e-mail between October and November 2020. The potential participants were reassured that nonparticipation would not have any consequences. A code was assigned to participants, prior to statistical analysis, thus guaranteeing the confidentiality of their data.

The inclusion criteria were that the participant needed to be a student at the Universidad Europea de Madrid ${ }^{34}$ and be between 18 and 34 years of age. ${ }^{41}$ The exclusion criteria were situations of: 1) having a chronic disease $;{ }^{42} 2$ ) undergoing pharmacological therapy, ${ }^{42}$ or 3 ) having any condition that led to development of pain or any disturbances during physical exercise. ${ }^{42}$

\section{Ethical considerations}

The current study was approved by the Research Ethics Committee of Universidad Europea de Madrid, under the protocol number CIPI/20/16, on September 30, 2020, and also respected the Helsinki guidelines at all times. All the participants read and signed an informed consent statement before becoming part of this investigation.

\section{Measurements}

- Physical activity level and sitting time were measured using the Global Physical Activity Questionnaire (WHO, Geneva, Switzerland) (GPAQ), a validated tool that was developed by WHO for estimating physical activity levels (MET-minutes/week) in diverse countries around the world. This questionnaire makes it possible to see whether the subjects are complying with the 2020 
WHO physical exercise recommendations, and whether they are spending large amounts of time seated. The amount of sitting time associated with greater all-cause mortality among adults varies from 6 to 8 hours per day, so we established $>420 \mathrm{~min}$ utes/day as the health risk threshold for this study.,8,43-45

- Adherence to the Mediterranean diet was measured using the PREDIMED questionnaire (Schröder et al., Barcelona, Spain).$^{43}$ High adherence is considered to be shown by scores $\geq 10$ points; medium adherence, 8-9 points; and low adherence $\leq 7$ points. Having high adherence to the Mediterranean diet brings strong protection against CVD. ${ }^{44}$

- HrQoL was measured using the short-form-36 (SF-36) health survey, version 2 (Alonso et al., Barcelona, Spain) ${ }^{45}$ which gives scores from 0 (worst health status) to 100 (best health status) in eight sections: physical function (PF), role physical (RP), bodily pain (BP), general health $(\mathrm{GH})$, vitality (VT), social functioning(SF), role emotional (RE) and mental health $(\mathrm{MH})$. The eight sections are regrouped into two main components: physical component summary (PCS) and mental component summary. ${ }^{41}$ The mean values for PCS and MCS are: 55.02 and 51.47 for men aged 18-24 years; 54.96 and 51.53 for men aged 25-34 years; 54.58 and 48.92 for women aged 18 - 24 years; and 53.87 and 49.62 for women aged $25-34$ years. ${ }^{41}$

- Body composition: height, weight and WC were measured using a stadiometer (in cm; Ano Sayol SL height rod, Barcelona, Spain), a scale (in kg; Asimed T2 scale, Barcelona, Spain) and a tape (in $\mathrm{cm}$ ), respectively. BMI and the waist-to-height ratio (WtHr) were then calculated. The values associated with an exponential risk of developing CVD and T2DM are WC $\geq 102$ $\mathrm{cm}$ in men and $\geq 88 \mathrm{~cm}$ in women, ${ }^{46}$ and $\mathrm{WtHr} \geq 0.5$ in men and women. ${ }^{47} \mathrm{WC}$ and $\mathrm{WtHr}$ are highly correlated with visceral adipose tissue, which is the most clinically relevant adiposity variable, because of its association with the increased risk of NCDs. In contrast, BMI has a poor correlation with visceral adipose tissue. ${ }^{47,48}$ However, individuals with high BMI are at greater risk of developing high visceral adipose tissue levels, compared with individuals with healthy BMI. ${ }^{49}$

\section{Statistical analysis}

Frequencies (sample size and proportion of samples) were assessed for categorical variables (adherence to Mediterranean diet and sitting time). The Kolmogorov-Smirnov test was used to assess the normality of distribution..$^{50}$ A descriptive analysis was developed for all the subjects using the mean \pm standard deviation (SD) to describe the parametric data and the median \pm interquartile range (IQR) for nonparametric data. The coefficient of variation (CV\%) was calculated for all continuous variables. In addition, an independent-sample t test (in situations of normal distribution) or the Mann-Whitney $\mathrm{U}$ test (in situations of non-normal distribution) was applied to determine differences between high/low adherence to the Mediterranean diet and the remainder of the continuous variables (HrQoL, physical activity and $\mathrm{BC}$ ).

The Spearman correlation test with 95\% coefficient intervals was carried out to analyze the relationships between continuous variables. The magnitudes of correlations between continuous variables were qualitatively interpreted using the following criteria: trivial $(r \leq 0.1)$, small $(r=0.1-0.3)$, moderate $(r=0.3-0.5)$, large $(r=0.5-0.7)$, very large $(r=0.7-0.9)$ and almost perfect $(r \geq 0.9) .{ }^{51}$ Otherwise, correlation was interpreted as the observed magnitude. The statistical significance was set at an alpha level of $<0.05$. All analyses were conducted using IBM SPSS for Windows, version 23 (IBM Corporation, Armonk, New York, United States).

\section{RESULTS}

\section{Sociodemographic data of the sample}

A total of 56 subjects aged $23.5 \pm 3.4$ years were analyzed. Their mean BMI was $22.15 \pm 2.28 \mathrm{~kg} / \mathrm{m}^{2}$. Regarding WC and WtHr, only three (5\%) and six subjects (10.7\%), respectively, had health-risk values.

The adherence to Mediterranean diet showed equality of distribution. Twenty-seven subjects (48.2\%) showed medium-high adherence while the remaining 29 subjects (51.8\%) showed low adherence. Analysis on sitting time revealed that 25 subjects were at risk $(44.6 \%)$ while the remainder of the subjects were at low risk ( $\mathrm{n}=31 ; 55.4 \%)$. Regarding HrQoL, 22 subjects (39.2\%) and 26 subjects (46.4\%) were in the lowest quintiles of PCS and MCS, respectively, according to the reference values for their age range.

The descriptive analysis, Kolmogorov-Smirnov normality test and $\mathrm{CV} \%$ for all the continuous variables is presented in Table 1.

\section{Correlations between body composition, lifestyle and HrQoL}

The Spearman correlation test with 95\% coefficient intervals was used to analyze possible correlations between continuous variables (Table 2$)$. Three HrQoL variables, namely VT $(\mathrm{P}=0.013)$, $\mathrm{RE}(\mathrm{P}=0.018)$ and MCS $(\mathrm{P}=0.015)$, showed moderate correlations (range for $r=0.31-0.33$ ) with WC. In turn, SF was moderately correlated $(r=0.27)$ with sitting time and there was a negative correlation between PF and sitting time $(r=-0.38$; moderate magnitude). In addition, trivial-small correlations (range for $r$ $=-0.15$ to 0.23 ) were found between HrQoL variables and the remainder of the physical activity and $\mathrm{BC}$ variables.

Between physical activity variables and the remainder of the continuous variables (BC and $\mathrm{HrQoL}$ ), trivial-small correlations (range for $r=-0.17$ to 0.24 ) were found. On the other hand, largevery large correlations (range for $r=0.56-0.82$ ) were found between $\mathrm{BC}$ variables (Table 2). 
Table 1. Descriptive analysis on all continuous variables

\begin{tabular}{|c|c|c|c|c|}
\hline & Variable $(n=56)$ & Value & $\mathrm{CV} \%$ & P-value of Kolmogorov-Smirnov test \\
\hline Body composition & Waist-to-height ratio & $0.43 \pm 0.04(0.35-0.59)^{\dagger}$ & 9 & 0.001 \\
\hline Physical activity & Sedentary time & $420.00 \pm 127.50(360.0-920.0)^{\dagger}$ & 26 & 0.000 \\
\hline \multirow{7}{*}{$\begin{array}{l}\text { Health-related quality } \\
\text { of life }\end{array}$} & Physical functioning & $100.00 \pm 5.00(50.0-100.0)^{\dagger}$ & 8 & 0.000 \\
\hline & Role physical & $100.00 \pm 43.80(0.0-100.0)^{\dagger}$ & 44 & 0.000 \\
\hline & Vitality & $66.07 \pm 17.12(20.0-100.0)^{*}$ & 26 & 0.070 \\
\hline & Social functioning & $87.50 \pm 25.00(37.5-100.0)^{\dagger}$ & 17 & 0.000 \\
\hline & Role emotional & $83.50 \pm 91.80(0.0-100.0)^{\dagger}$ & 71 & 0.000 \\
\hline & Mental health & $80.00 \pm 20.00(20.0-100.0)^{\dagger}$ & 19 & 0.003 \\
\hline & Physical component summary & $53.16 \pm 7.15(35.4-67.4)^{*}$ & 13 & 0.200 \\
\hline
\end{tabular}

Values shown are ${ }^{*}$ mean \pm standard deviation (SD) (minimum-maximum) or $^{\dagger}$ median \pm interquartile range (IQR) (minimum-maximum). CV\% $=$ coefficient of variation.

Table 2. Relationship (Spearman correlation test) between continuous variables with 95\% coefficient intervals

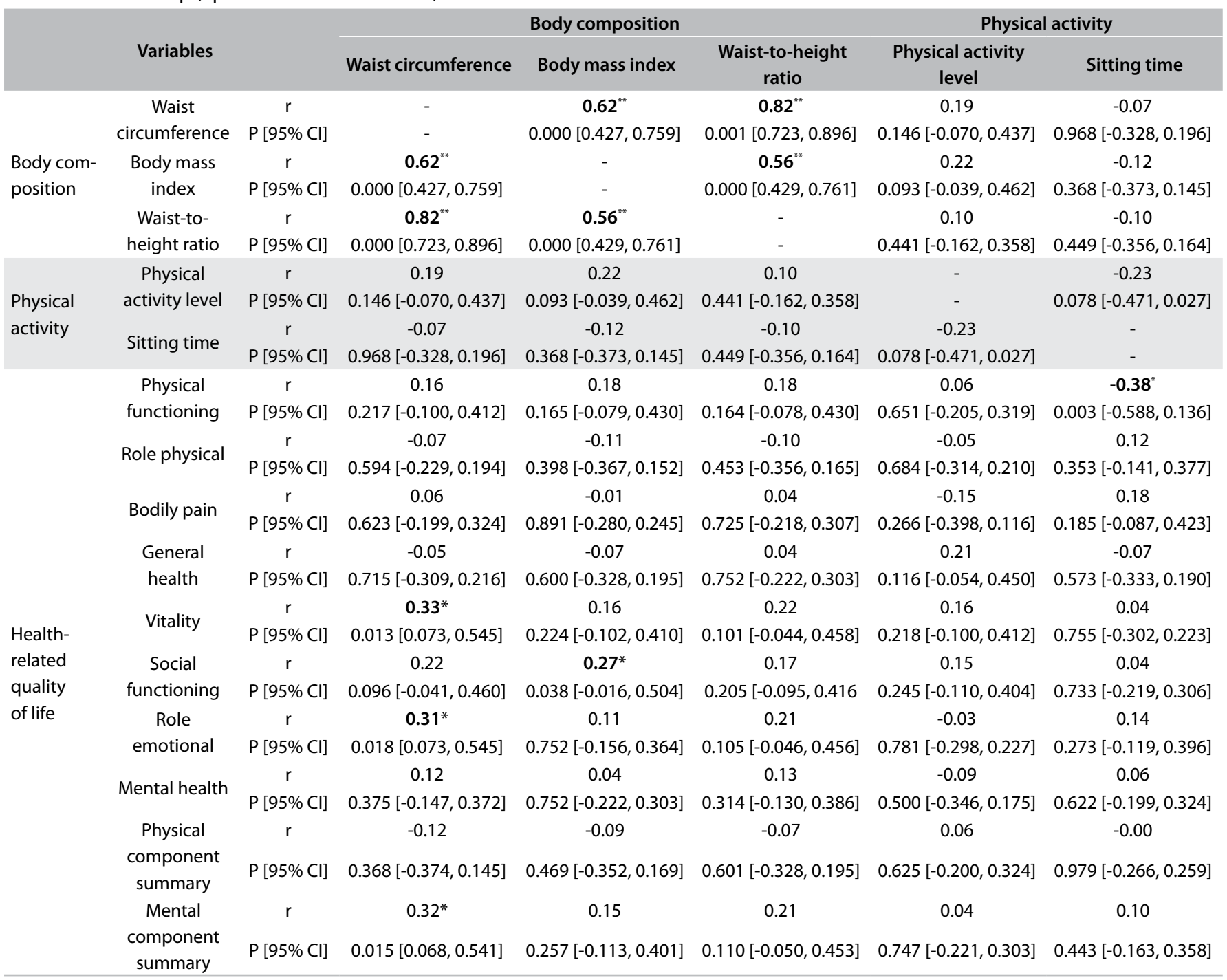


Nonsignificant differences in BC, physical activity and HrQoL were found between medium-high and low adherence to the Mediterranean diet (Table 3). The comparisons of BC, physical activity and HrQoL between risk and non-risk sitting times are displayed in Table 4.

\section{DISCUSSION}

The subjects analyzed in this study had healthy BC values, as only $5 \%$ and $10.7 \%$ of the subjects had health-risk values for WC and WtHr, respectively. Regarding lifestyle, $44.6 \%$ of the subjects spent more than seven hours a day sitting down, which is a predisposing factor for development of cardiometabolic diseases over the long term. ${ }^{24,52}$ Individuals who maintain sedentary behavior over time have the highest all-cause mortality. ${ }^{8}$ Levels of physical activity were high (1880.37 $\pm 738.90 \mathrm{MET}$-min/week), such that $92.86 \%$ of the subjects were complying with the WHO 2020 physical activity recommendations. ${ }^{53}$ This was similar to the results reported in other studies with a student population. ${ }^{36,37}$

Physical activity attenuates mortality risk, and higher physical activity levels are required among highly sedentary individuals. ${ }^{8}$

Table 3. Differences in body composition, physical activity and health-related quality of life between medium-high and low adherence to the Mediterranean diet

\begin{tabular}{|c|c|c|c|c|}
\hline & Variable & $\begin{array}{l}\text { Medium-high adherence group (n } \\
\qquad=27 \text { ) }\end{array}$ & Low adherence group $(n=29)$ & $P$ value \\
\hline \multirow[b]{2}{*}{ Body composition } & Waist circumference $(\mathrm{cm})$ & $76.00 \pm 8.00(67.0-92.0)^{\dagger}$ & $76.00 \pm 12.50(65.0-102.0)^{\dagger}$ & $0.967^{\ddagger}$ \\
\hline & Body mass index (\%) & $22.07 \pm 2.17(18.6-26.8)^{*}$ & $22.22 \pm 2.41(19.0-26.9)^{*}$ & 0.820 \\
\hline \multirow{2}{*}{ Physical activity } & Physical activity level (MET-min/week) & $2011.85 \pm 707.89(800.0-3360.0)^{*}$ & $1757.93 \pm 756.33(240.0-3440.0)^{*}$ & 0.201 \\
\hline & Sitting time (minutes/week) & $420.00 \pm 60.00(360.0-920.0)^{\dagger}$ & $480.00 \pm 180.00(420.0-900.0)^{\dagger}$ & $0.195^{\ddagger}$ \\
\hline \multirow{5}{*}{$\begin{array}{l}\text { Health-related } \\
\text { quality of life }\end{array}$} & Bodily pain & $84.00 \pm 22.00(20.0-100.0)^{+}$ & $84.00 \pm 22.00(32.0-100.0)^{\dagger}$ & $0.839^{\ddagger}$ \\
\hline & General health & $73.88 \pm 17.42(30.0-97.0)^{*}$ & $71.37 \pm 15.94(42.0-97.0)^{*}$ & 0.576 \\
\hline & Vitality & $66.48 \pm 21.29(20.0-100.0)^{*}$ & $65.69 \pm 12.44(45.0-85.0)^{*}$ & 0.865 \\
\hline & Social functioning & $87.50 \pm 25.80(37.5-100.0)^{\dagger}$ & $87.50 \pm 18.80(62.5-100.0)^{\dagger}$ & $0.473^{\ddagger}$ \\
\hline & Role emotional & $100.00 \pm 100.00(00.0-100.0)^{\dagger}$ & $66.70 \pm 83.50(00.0-100.0)^{\dagger}$ & $0.677^{\ddagger}$ \\
\hline
\end{tabular}

Comparisons are between high $(n=27)$ and low $(n=29)$ adherence to the Mediterranean diet. ${ }^{*}$ Mean \pm standard deviation (SD) (minimum-maximum) or ${ }^{\dagger}$ Median \pm interquartile range (IR) (minimum-maximum). Statistical significance was set at an alpha level of $<0.05\left(^{*}\right)$. ${ }^{*}$ Mann-Whitney $\mathrm{U}$ test was used.

Table 4. Differences in body composition, physical activity and health-related quality of life between risk and non-risk sitting time

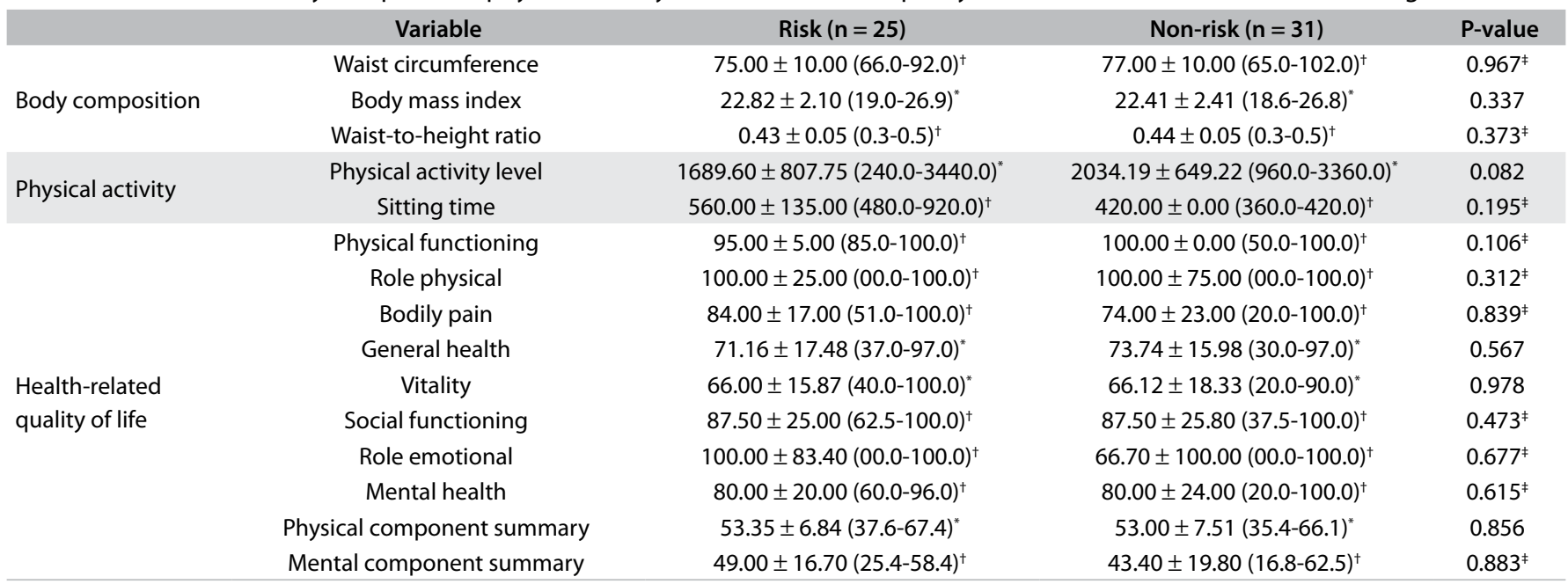

Comparisons are between risk $(n=25)$ and non-risk $(n=31)$ sitting time. ${ }^{*}$ Mean \pm standard deviation (SD) (minimum-maximum) or ${ }^{\dagger}$ median \pm interquartile range (IQR) (minimum-maximum). Statistical significance was set at an alpha level of $<0.05\left(^{*}\right)$. ${ }^{\star}$ Mann-Whitney $U$ test was used. 
Each additional hour of sedentary time is associated with greater gain in BMI and WC. ${ }^{8}$ However, in this study, we found only trivial-small correlations for physical activity and sitting time in relation to BC. The mean daily sitting time was 8.26 hours, while $19.64 \%$ of the subjects spent $\geq 10$ hours per day sitting. ${ }^{8}$ This is of great importance because if these habits are maintained over time, the risk of developing NCDs increases, ${ }^{3}$ given that sedentary behavior is associated with loss of metabolic flexibility, ${ }^{3}$ higher oxidative stress, insulin resistance, inflammation and DNA damage. ${ }^{54,55}$ Self-reported estimates from other studies have indicated that university students spend an average of 7.29 hours per day sitting, which is similar to our results. In contrast, data from accelerometer-based studies have suggested that university students engage in 9.82 hours of sedentary behavior per day. Chastin et al. suggested that self-reports underestimate sedentary behavior, compared with accelerometer-based methods, ${ }^{37}$ so the subjects of our study may have been spending more time sitting that we thought.

Concerning nutritional habits, half of the subjects analyzed (51.8\%) had low adherence to the Mediterranean diet. The mean PREDIMED score was 7.41, which denotes acceptable adherence to the Mediterranean diet, in line with data from the Spanish population that was previously reported. ${ }^{26}$ However, other studies on student populations reported lower adherence to the Mediterranean diet. ${ }^{34,35}$ Considering these data, it is necessary to seek strategies to reduce sitting time in the young population, in addition to promoting the Mediterranean diet, since the lifestyle habits developed in youth can affect health in old age. It was striking that $39.2 \%$ and $46.4 \%$ of the subjects were in the lowest quintiles of PCS and MCS, respectively, according to the reference values for their age range. ${ }^{41}$

We did not find any study reporting on HrQoL using the SF-36 questionnaire among students that we could compare with our results. However, several authors have argued that the negative impact on QoL among sedentary individuals and university students was related to foot health disorders. ${ }^{56,57}$ The low levels of HrQoL reported in the present study are probably related to the massive reorganization of our society and our lifestyle due to the COVID-19 pandemic. ${ }^{29}$

Regarding the relationship between $\mathrm{BC}$ and lifestyle variables and HrQoL, WC showed moderate correlations with VT, RE and MCS. This is not of great clinical relevance because most of the subjects had healthy values for WC. In turn, sitting time was negatively correlated with $\mathrm{PF}$, which highlights the negative impact of sedentary behavior on health. ${ }^{7}$ However, taking $>7$ hours of sitting time per day as a reference value for health risk, no significant correlations were found between sitting time and the other variables. Adherence to the Mediterranean diet did not show any significant correlation with $\mathrm{HrQoL}$ or BC, which is relevant because this young population may not see any motivation to engage in changes in this regard. Moreover, large correlations exist between $\mathrm{WC}$ and $\mathrm{BMI}$, and very large correlations exist between WC and WtHr. It is known that WC is more associated with cardiometabolic diseases risk factors, as well as $\mathrm{WtHr},{ }^{58}$ compared with BMI. In fact, in other populations, WC is a better indicator of poor physical HrQoL than BMI. ${ }^{59}$

The aim of this study was to characterize the lifestyle of young students in a pandemic period. We mainly found that these subjects were complying with the 2020 WHO physical exercise recommendations ${ }^{53}$ and had healthy BC. However, half of them had a poor dietary pattern and spent too many hours sitting. According to several studies, during the current COVID19 pandemic period, physical activity levels have decreased by $30 \%{ }^{30}$ and daily sitting time has increased (28.6\%), ${ }^{31}$ while adherence to the Mediterranean diet has not been affected. ${ }^{32}$ This indicates that there needs to be a focus on improving these two aspects of the lifestyle of the young population. We also wanted to analyze $\mathrm{HrQoL}$ and its correlations with lifestyle. Sitting time was the variable that most negatively affected HrQoL, and it was striking that $39.2 \%$ and $46.4 \%$ of the subjects were in the lowest quintiles of PCS and MCS, respectively, according to the reference values for their age range. These results might be related to the difficult times of uncertainty and restriction of mobility that we have been going through, in the context of the COVID-19 pandemic.

\section{Study limitations and future lines}

Our sample was obtained from just one particular university in one particular city. It would be very useful to obtain information from more universities in different locations. In addition, studying the implementation of a strengthening program or physical activity in relation to the Mediterranean diet, both among healthy individuals and among individuals presenting any pathological condition (e.g. COVID-19, musculoskeletal disorders or metabolic diseases) would be very interesting. Lastly, no comparative data from before the pandemic were obtained and, thus, the PCS and MCS cannot be attributed to the COVID-19 pandemic.

\section{CONCLUSION}

In this young student population, the results showed that the subjects generally had healthy BC, high physical activity levels, acceptable adherence to the Mediterranean diet, high levels of sedentary behavior and very low levels of HrQoL. Regarding the relationship between lifestyle and HrQoL, only trivial-small correlations were found. Regarding lifestyle, the findings of the present study especially highlight the importance of implementation of public health programs targeting reductions in sitting time among university students. 


\section{REFERENCES}

1. Schwingshackl L, Morze J, Hoffmann G. Mediterranean diet and health status: Active ingredients and pharmacological mechanisms. Br J Pharmacol. 2020;177(6):1241-57. PMID: 31243760; https://doi. org/10.1111/bph.14778.

2. Mak YW, Kao AHF, Tam LWY, et al. Health-promoting lifestyle and quality of life among Chinese nursing students. Prim Heal Care Res Dev. 2018;19(6):629-36. PMID: 29623871; https://doi.org/10.1017/ S1463423618000208.

3. Arocha Rodulfo JI. Sedentarism, a disease from XXI century. Clin Investig Arterioscler. 2019;31(5):233-40. PMID: 31221536; https://doi. org/10.1016/j.arteri.2019.04.004.

4. WHO. Global recommendations on physical activity for health. Geneva: WHO Press, World Health Organization; 2010. Available from: https:// www.who.int/publications/i/item/9789241599979. Accessed in 2021 (May 11).

5. Global action plan on physical activity 2018-2030: more active people for a healthier world. Geneva: World Health Organization; 2018. Available from: https://apps.who.int/iris/bitstream/hand le/10665/272722/9789241514187-eng.pdf. Accessed in 2021 (Mar 19).

6. Booth FW, Roberts CK, Thyfault JP, Ruegsegger GN, Toedebusch RG. Role of Inactivity in Chronic Diseases: Evolutionary Insight and Pathophysiological Mechanisms. Physiol Rev. 2017;97(4):1351-402. PMID: 28814614; https://doi.org/10.1152/physrev.00019.2016.

7. Matthews CE, Keadle SK, Troiano RP, et al. Accelerometer-measured dose-response for physical activity, sedentary time, and mortality in US adults. Am J Clin Nutr. 2016;104(5):1424-32. PMID: 27707702; https:// doi.org/10.3945/ajcn.116.135129.

8. Katzmarzyk PT, Powell KE, Jakicic JM, et al. Sedentary Behavior and Health: Update from the 2018 Physical Activity Guidelines Advisory Committee. Med Sci Sports Exerc. 2019;51 (6):1227-41. PMID: 31095080; https://doi.org/10.1249/MSS.0000000000001935.

9. Aboonabi A, Meyer RR, Singh I. The association between metabolic syndrome components and the development of atherosclerosis. J Hum Hypertens. 2019;33(12):844-55. PMID: 31636352; https://doi. org/10.1038/s41371-019-0273-0.

10. Schäffler A, Schölmerich J, Büchler C. Mechanisms of disease: adipocytokines and visceral adipose tissue--emerging role in intestinal and mesenteric diseases. Nat Clin Pract Gastroenterol Hepatol. 2005;2(2):103-11. PMID: 16265128; https://doi.org/10.1038/ ncpgasthep0090.

11. Eckardt K, Görgens SW, Raschke S, Eckel J. Myokines in insulin resistance and type 2 diabetes. Diabetologia. 2014;57(6):1087-99. PMID: 24676645; https://doi.org/10.1007/s00125-014-3224-x.

12. Díaz BB, González DA, Gannar F, Pérez MCR, de León AC. Myokines, physical activity, insulin resistance and autoimmune diseases. Immunol Lett. 2018;203:1-5. PMID: 30194964; https://doi.org/10.1016/j. imlet.2018.09.002.
13. Blüher M. Obesity: global epidemiology and pathogenesis. Nat Rev Endocrinol. 2019;15(5):288-98. PMID: 30814686; https://doi.org/10.1038/ s41574-019-0176-8.

14. Karczewski J, Śledzińska E, Baturo A, et al. Obesity and inflammation. Eur Cytokine Netw. 2018;29(3):83-94. PMID: 30547890; https://doi. org/10.1684/ecn.2018.0415.

15. Reilly SM, Saltiel AR. Adapting to obesity with adipose tissue inflammation. Nat Rev Endocrinol. 2017;13(11):633-43. PMID: 28799554; https://doi.org/10.1038/nrendo.2017.90.

16. Asghar A, Sheikh N. Role of immune cells in obesity induced low grade inflammation and insulin resistance. Cell Immunol. 2017;315:18-26. PMID: 28285710; https://doi.org/10.1016/j.cellimm.2017.03.001.

17. Saltiel AR. Insulin resistance in the defense against obesity. Cell Metab. 2012;15(6):798-804. PMID: 22682220; https://doi.org/10.1016/j. cmet.2012.03.001.

18. Avgerinos Kl, Spyrou N, Mantzoros CS, Dalamaga M. Obesity and cancer risk: Emerging biological mechanisms and perspectives. Metabolism. 2019 Mar;92:121-135. PMID: 30445141; https://doi.org/10.1016/j. metabol.2018.11.001.

19. Weber Buchholz S, Wilbur J, Halloway S, McDevitt JH, Schoeny ME. Physical activity intervention studies and their relationship to body composition in healthy women. Annu Rev Nurs Res. 2013;31:71-142. PMID: 24894138; https://doi.org/10.1891/0739-6686.31.71.

20. Welc SS, Clanton TL. The regulation of interleukin- 6 implicates skeletal muscle as an integrative stress sensor and endocrine organ. Exp Physiol. 2013;98(2):359-71. PMID: 22941979; https://doi.org/10.1113/ expphysiol.2012.068189.

21. Nimmo MA, Leggate M, Viana JL, King JA. The effect of physical activity on mediators of inflammation. Diabetes Obes Metab. 2013;15 Suppl 3:51-60. PMID: 24003921; https://doi.org/10.1111/dom.12156.

22. Wedell-Neergaard AS, Krogh-Madsen R, Petersen GL, et al. Cardiorespiratory fitness and the metabolic syndrome: Roles of inflammation and abdominal obesity. PLoS One. 2018;13(3):e0194991. PMID: 29590212; https://doi.org/10.1371/journal.pone.0194991.

23. Pedersen BK. Anti-inflammatory effects of exercise: role in diabetes and cardiovascular disease. Eur J Clin Invest. 2017;47(8):600-11. PMID: 28722106; https://doi.org/10.1111/eci.12781.

24. Tsigalou C, Konstantinidis T, Paraschaki A, et al. Mediterranean Diet as a Tool to Combat Inflammation and Chronic Diseases. An Overview. Biomedicines. 2020;8(7):201. PMID: 32650619; https://doi.org/10.3390/ biomedicines 8070201

25. Bendall CL, Mayr HL, Opie RS, et al. Central obesity and the Mediterranean diet: A systematic review of intervention trials. Crit Rev Food Sci Nutr. 2018;58(18):3070-84. PMID: 29039967; https://doi.org/10.1080/10408 398.2017.1351917.

26. Ramón-Arbués E, Martínez-Abadía B, Granada-López JM, et al. Association between adherence to the Mediterranean diet and the prevalence of cardiovascular risk factors. Rev Lat Am Enfermagem. 2020;28:e3295. PMID: 32520245; https://doi.org/10.1590/1518-8345.3904.3295. 
27. Wu F, Zhao S, Yu B, et al. A new coronavirus associated with human respiratory disease in China. Nature. 2020;579(7798):265-9. PMID: 32015508; https://doi.org/10.1038/s41586-020-2008-3. Erratum in: Nature. 2020 Apr;580(7803):E7.

28. Velavan TP, Meyer CG. The COVID-19 epidemic. Trop Med Int Health. 2020;25(3):278-80. PMID: 32052514; https://doi.org/10.1111/tmi.13383.

29. Wijesooriya NR, Mishra V, Brand PLP, Rubin BK. COVID-19 and telehealth, education, and research adaptations. Paediatr Respir Rev. 2020;35:38-42. PMID: 32653468; https://doi.org/10.1016/j.prrv.2020.06.009.

30. Castañeda-Babarro A, Arbillaga-Etxarri A, Gutiérrez-Santamaría B, Coca A. Physical Activity Change during COVID-19 Confinement. Int J Environ Res Public Health. 2020;17(18):6878. PMID: 32967091; https://doi. org/10.3390/ijerph17186878.

31. Patterson R, McNamara E, Tainio M, et al. Sedentary behaviour and risk of all-cause, cardiovascular and cancer mortality, and incident type 2 diabetes: a systematic review and dose response meta-analysis. Eur J Epidemiol. 2018;33(9):811-29. PMID: 29589226; https://doi.org/10.1007/ s10654-018-0380-1.

32. Sánchez-SánchezE, Ramírez-Vargas G, Avellaneda-LópezY, et al. Eating Habits and Physical Activity of the Spanish Population during the COVID-19 Pandemic Period. Nutrients. 2020;12(9):2826. PMID: 32942695; https://doi.org/10.3390/nu12092826.

33. Zaragoza-Martí A, Cabañero-Martínez MJ, Hurtado-Sánchez JA, Laguna-Pérez A, Ferrer-Cascales R. Evaluation of Mediterranean diet adherence scores: a systematic review. BMJ Open. 2018;8(2):e019033. PMID: 29478018; https://doi.org/10.1136/bmjopen-2017-019033.

34. Cobo-Cuenca Al, Garrido-Miguel M, Soriano-Cano A, et al. Adherence to the Mediterranean Diet and Its Association with Body Composition and Physical Fitness in Spanish University Students. Nutrients. 2019;1 1(11):2830. PMID:31752296; https://doi.org/10.3390/nu11112830.

35. Antonopoulou M, Mantzorou M, Serdari A, et al. Evaluating Mediterranean diet adherence in university student populations: Does this dietary pattern affect students' academic performance and mental health? Int J Health Plann Manage. 2020;35(1):5-21. PMID: 31514237; https:// doi.org/10.1002/hpm.2881.

36. Peterson NE, Sirard JR, Kulbok PA, DeBoer MD, Erickson JM. Sedentary behavior and physical activity of young adult university students. Res Nurs Health. 2018;41(1):30-8. PMID: 29315656; https://doi.org/10.1002/ nur.21845

37. Castro O, Bennie J, Vergeer I, Bosselut G, Biddle SJH. How Sedentary Are University Students? A Systematic Review and Meta-Analysis. Prev Sci. 2020;21(3):332-43. PMID: 31975312; https://doi.org/10.1007/ s11121-020-01093-8.

38. Vankim NA, Nelson TF. Vigorous physical activity, mental health, perceived stress, and socializing among college students. Am J Health Promot. 2013;28(1):7-15. PMID: 23470187; https://doi.org/10.4278/ ajhp.111101-QUAN-395.

39. Snedden TR, Scerpella J, Kliethermes SA, et al. Sport and Physical Activity Level Impacts Health-Related Quality of Life Among Collegiate Students.
Am J Health Promot. 2019;33(5):675-82. PMID: 30586999; https://doi. org/10.1177/0890117118817715

40. Nowak PF, Bożek A, Blukacz M. Physical Activity, Sedentary Behavior, and Quality of Life among University Students. Biomed Res Int. 2019;2019:9791281. PMID: 31930143; https://doi. org/10.1155/2019/9791281.

41. Vilagut $G$, Valderas JM, Ferrer M, et al. Interpretación de los cuestionarios de salud SF-36 y SF-12 en España: Componentes físico y mental [Interpretation of SF-36 and SF-12 questionnaires in Spain: physical and mental components]. Med Clin (Barc). 2008;130(19):726-35. PMID: 18570798; https://doi.org/10.1157/13121076.

42. Úbeda-Colomer J, Devís-Devís J, Sit CHP. Barriers to physical activity in university students with disabilities: Differences by sociodemographic variables. Disabil Health J. 2019;12(2):278-86. PMID: 30446481; https:// doi.org/10.1016/j.dhjo.2018.11.005

43. Schröder H, Fitó M, Estruch R, et al. A short screener is valid for assessing Mediterranean diet adherence among older Spanish men and women. J Nutr. 2011 Jun;141(6):1140-5. PMID: 21508208; https://doi.org/10.3945/ jn.110.135566.

44. Martínez-González MA, García-Arellano A, Toledo E, et al. A 14-item Mediterranean diet assessment tool and obesity indexes among highrisk subjects: the PREDIMED trial. PLoS One. 2012;7(8):e43134. PMID: 22905215; https://doi.org/10.1371/journal.pone.0043134.

45. Alonso J, Prieto L, Antó JM. La versión española del SF-36 Health Survey (Cuestionario de Salud SF-36): un instrumento para la medida de los resultados clínicos. Med Clin. 1995;104(20):771-6. Available from: https:/www.uv.es/ docmed/documed/documed/705.html. Accessed in 2021 (May 12).

46. Zimmet P, M M Alberti KG, Serrano Ríos M. Una nueva definición mundial del síndrome metabólico propuesta por la federación Internacional de Diabetes: fundamento y resultados [A new international diabetes federation worldwide definition of the metabolic syndrome: the rationale and the results]. Rev Esp Cardiol. 2005;58(12):1371-6. PMID: 16371194; https://doi.org/10.1016/S0300-8932(05)74065-3. Erratum in: Rev Esp Cardiol. 2006;59(2):185

47. Swainson MG, Batterham AM, Tsakirides C, Rutherford ZH, Hind K. Prediction of whole-body fat percentage and visceral adipose tissue mass from five anthropometric variables. PLoS One. 2017;12(5):e0177175. PMID: 28493988; https://doi.org/10.1371/journal.pone.0177175.

48. Leggio M, Lombardi M, Caldarone E, Mazza A, Fusco A. High body mass index, healthy metabolic profile and low visceral adipose tissue: The paradox is to call it obesity again. Eur J Intern Med. 2018;52:e15-e16. PMID: 29636273; https://doi.org/10.1016/j.ejim.2018.03.019.

49. Kang YM, Jung $\mathrm{CH}$, Cho YK, et al. Visceral adiposity index predicts the conversion of metabolically healthy obesity to an unhealthy phenotype. PLoS One. 2017;12(6):e0179635. PMID: 28644850; https:// doi.org/10.1371/journal.pone.0179635.

50. Ghasemi A, ZahediasI S. Normality tests for statistical analysis: a guide for non-statisticians. Int J Endocrinol Metab. 2012 Spring;10(2):486-9. PMID: 23843808; https://doi.org/10.5812/ijem.3505. 
51. Hopkins WG, Marshall SW, Batterham AM, Hanin J. Progressive statistics for studies in sports medicine and exercise science. Med Sci Sports Exerc. 2009;41(1):3-13. PMID: 19092709; https://doi.org/10.1249/ MSS.0b013e31818cb278.

52. Matthews CE, George SM, Moore SC, et al. Amount of time spent in sedentary behaviors and cause-specific mortality in US adults. Am J Clin Nutr. 2012;95(2):437-45. PMID: 22218159; https://doi.org/10.3945/ ajcn.111.019620.

53. Bull FC, Al-Ansari SS, Biddle S, et al. World Health Organization 2020 guidelines on physical activity and sedentary behaviour. Br J Sports Med. 2020;54(24):1451-62. PMID: 33239350; https://doi.org/10.1136/ bjsports-2020-102955.

54. Navarro-Ibarra MJ, Hernández J, Caire-Juvera G. Diet, physical activity and telomere length in adults. Nutr Hosp. 2019;36(6):1403-17. PMID: 31657606; https://doi.org/10.20960/nh.02673.

55. Pratesi A, Tarantini F, Di Bari M. Skeletal muscle: an endocrine organ. Clin Cases Miner Bone Metab. 2013;10(1):11-4.PMID: 23858303; https:// doi.org/10.11138/ccmbm/2013.10.1.011.

56. López-López D, Cancela-Carral JM, Araujo R, et al. Association between sex differences on foot health related to the quality of life in a sample of sedentary people. Rev Assoc Med Bras (1992). 2019;65(2):149-55. PMID: 30892437; https://doi.org/10.1590/1806-9282.65.2.149.

57. Rodríguez-Sanz D, Barbeito-Fernández D, Losa-Iglesias ME, et al. Foot health and quality of life among university students: cross-sectional study. Sao Paulo Med J. 2018;136(2):123-8. PMID: 29617469; https:// doi.org/10.1590/1516-3180.2017.0264230917.

58. Jabłonowska-Lietz B, Wrzosek M, Włodarczyk M, Nowicka G. New indexes of body fat distribution, visceral adiposity index, body adiposity index, waist-to-height ratio, and metabolic disturbances in the obese. Kardiol Pol. 2017;75(11):1185-91. PMID: 28715064; https://doi.org/10.5603/ KP.a2017.0149.

59. Hyun YY, Lee KB, Chung W, et al. Body Mass Index, waist circumference, and health-related quality of life in adults with chronic kidney disease. Qual Life Res. 2019;28(4):1075-83. PMID: 30535570; https://doi. org/10.1007/s11136-018-2084-0.

Authors' contributions: Pérez de Sevilla GG: investigation (equal), methodology (equal) and writing-original draft (equal); Chao EAP: formal analysis (equal), investigation (equal), project administration (equal), software (equal) and writing-review and editing (equal); Galeano HP: methodology (equal), supervision (equal) and writing-review and editing (equal); Martinez-Jimenez EM: methodology (equal), review and editing (equal); San Frutos MP: data curation (equal), investigation (equal) and methodology (equal); Pinto BSP: investigation (equal), methodology (equal) and writing-original draft (equal); and Morales CR: formal analysis (equal), investigation (equal) and writing-review and editing (equal). All authors actively contributed to the discussion of the results from study, reviewed and approved the final version to be released

\section{Sources of funding: None}

Conflict of interest: None

Date of first submission: February 20, 2021

Last received: March 9, 2021

Accepted: March 12, 2021

\section{Address for correspondence:}

Carlos Romero Morales

Universidad Europea de Madrid

Calle Tajo, s/no, 28.670, Villaviciosa de Odón, Madrid, Spain

Tel. +34 917407272

E-mail: carlosmorales92@hotmail.com 